\title{
A new scheme to calculate isotope effects
}

\author{
Katarzyna Świderek • Agnieszka Dybala-Defratyka • \\ Daniel R. Rohr
}

Received: 7 September 2010 / Accepted: 1 October 2010/Published online: 19 October 2010

(C) The Author(s) 2010. This article is published with open access at Springerlink.com

\begin{abstract}
We present a new scheme to calculate isotope effects. Only selected frequencies at the target level of theory are calculated. The frequencies are selected by an analysis of the Hessian from a lower level of theory. We obtain accurate isotope effects without calculating the full Hessian at the target level of theory. The calculated frequencies are very accurate. The scheme converges to the correct isotope effect.
\end{abstract}

Keywords Computational chemistry · Isotope effects . Molecular modeling

\section{Introduction}

In catalysis isotope effects have been used to identify the reaction mechanism and understand the interactions between the ligands and the receptor [1]. Two chemically identical systems are investigated, which differ only by the mass of one or more atoms. The two systems are called isotopologes. The kinetic isotope effect is the ratio of reaction rates of the two isotopologes. It can provide unique information about the structure of the transition state, which plays an important role in distinguishing between possible mechanisms. Equilibrium isotope effects are calculated

\footnotetext{
K. Świderek · A. Dybala-Defratyka

Faculty of Chemistry, Institue of Applied Radiation Chemistry,

Technical University of Łódź,

Ulica Żeromskiego 116,

90-924 Łódź, Poland

\section{R. Rohr $(\bowtie)$}

Institue of Physics, Technical University of Łódź,

Ulica Wólczańska 219,

93-005 Łódź, Poland

e-mail: rohrdr@gmail.com
}

from the ratio of the equilibrium constants. This analysis turned out to be a useful tool for describing atomic interactions between reactants and the catalytic sites during the binding step of the catalysis [2]. Isotope effects are experimentally accessible using different techniques and methods [3]. If a reaction is composed of several steps, which is usually the case in enzymatic processes, a complementary tool is needed to interpret and understand the experimental data.

The observed isotope effect is the net effect, a weighted sum of each of the separate steps. Computational tools have the power to provide information about each of the steps. They can be used to predict the isotope effect for an assumed reaction mechanism or binding mechanism. The results can then be compared with experimental data. Modeling of enzymatic processes is a challenging task. Compromises on the way from model preparation to the calculation of the isotope effect is required. The most commonly used scheme is the quantum mechanics/molecular mechanics (QM/MM) hybrid method. Here, the reaction center is treated quantum mechanically, using either DFT or semiempirical methods. The rest of the system is treated classically, using available force fields.

Theoretical predictions of isotope effects date back as early as the 1960s. Based on the isotope effects theory of Bigeleisen and Goeppert-Mayer [4] model calculations using so-called cutoff models were proposed by Wolfsberg and Stern [5] and later by Sims and coworkers [6]. Due to the phenomenon that isotope effects depend on structure and bonding changes primarily in the regions of molecule close to the isotopically-substituted position Stern and Wolfsberg validated their procedure [7] for various cyclic and acyclic structures [5]. The initial studies used bond orders and tabulated force constants to describe isotope effects. The model was refined by the use of molecular 
mechanics to generate force fields and the inclusion of bond energies in the model. The new method was implemented in the BEBOVIB program [8]. The software predicts kinetic isotope effects based on the structure of the reactant and the transition state of a reaction. Different protocols were proposed for creating an appropriate transition state model [9-11]. However user-defined force constants and bond orders were often the source of apparent failure of the method.

Along with the technological boom in computer science observed over the last decades more reliable quantum mechanical calculations became feasible. Electronic structure calculations as well as algorithms for transition state location started to replace the BEBOVIB method. A number of studies have appeared showing discrepancies between the results obtained from BEBOVIB and quantum calculations [12-14].

Using the Teller-Redlich rules [15] the equilibrium or kinetic isotope effect can be solely calculated from the wave numbers of the reactant and products. This equation is known as the Bigeleisen-Mayer equation [16].

$I E=I F\left(\left\{v_{i, L}^{R}\right\},\left\{v_{i, H}^{R}\right\}\right) \cdot I F^{-1}\left(\left\{v_{i, L}^{P}\right\},\left\{v_{i, H}^{P}\right\}\right)$.

It is a product of the isotope factor (IF) for the reactant $(\mathrm{R})$ and the inverse of the IF of the product $(\mathrm{P})$. The term isotope factor (IF) is introduced in this paper, because we focus on only one factor without calculating the full isotope effect. The rationale is that if we can calculate the IF of, say, the reactant we can also calculate the IF of the product or transition state, since the formula is identical. The isotope factor is calculated from the wave numbers of the light isotopologe $\left(\left\{\nu_{i, L}\right\}\right)$ and the heavy isotopologe $\left(\left\{\nu_{i, H}\right\}\right)$ according to

$I F\left(\left\{v_{i, L}\right\},\left\{v_{i, H}\right\}\right)=\Pi \frac{v_{i, H} \cdot \sinh \left[v_{i, L} h /(2 k T)\right]}{v_{i, L} \cdot \sinh \left[v_{i, H} h /(2 k T)\right]}$.

Here $h$ is the Planck constant, $k$ is the Boltzman constant and $T$ is the temperature in Kelvin. Only in the case of the kinetic isotope effect the IF is slightly different. For the imaginary frequency of the transition state the sinh of the wave numbers are dropped. This means that only a factor of $\frac{v_{T S, H}}{V_{T S . L}}$ appears, where the $\mathrm{n} T S$ is the absolute value of the imaginary frequency.

When analyzing Eq. 2 we realize that the factors in the product assume the value one, if the wave number of the light and heavy isotopologe are equal. This is often the case, since only a few wave numbers are affected, if one or a few atoms are replaced by their heavier isotope. As a consequence, only a small number of wave numbers contribute to the isotope factor, and, hence, to the isotope effect.
Wave numbers are calculated from the eigenvalues of the matrix of second derivatives, also known as Hessian. The calculation of the Hessian is a time consuming task when enzymatic reactions with thousands of atoms are investigated. Moreover, QM/MM schemes usually do not allow for the analytic calculation of the Hessian. Consequently, the Hessian is calculated by the finite difference method. This makes the evaluation of the full Hessian practically impossible. Currently, an approximation to calculate the isotope effect in enzymatic reactions is used. A set of atoms is chosen, usually the atoms in the QM part. Then the second derivatives are calculated for only these atoms. These second derivatives form only part of the full Hessian, hence the name partial Hessian approach. Diagonalization of the partial Hessian gives approximate vibrations and wave numbers. From the approximate wave numbers approximate isotope factors and isotope effects are calculated.

When calculating the isotope effect two approximations are made. The first is the chosen level of theory. This is true for any kind of calculation and always needs to be addressed. The second approximation is the calculation of the Hessian. In this paper we address the latter approximation. We propose a new scheme to calculate the isotope factor for a target level of theory. We selectively calculate only those wave numbers that contribute to the IF. We do this by first identifying the contributing wave numbers from an analysis of the Hessian from a lower level of theory. The corresponding vibrational modes of the identified wave numbers are then used to calculate the contributing wave numbers at the target level of theory. The details of our scheme are explained in section Theory.

In Section Results we show the results obtained with the new scheme for a test system. We show that our scheme converges to the right isotope factor from below. Our scheme gives accurate wave numbers. We also compare our scheme to the partial Hessian approach. In Section Conclusions we draw the conclusions. We find that the partial Hessian approach is superior to our scheme.

\section{Theory}

In this Section we explain the details of the new scheme. We start by reviewing some basic terminology and their definitions in Subsection Terminology and definitions. The new scheme allows us to calculate only selected frequencies. To this end the Hessian is projected onto the space spanned by the selected frequencies. In Subsection Projected Hessian we review the equations for the projection of the Hessian. In Subsection Calculation of selected frequencies we explain how to calculate the projected Hessian without calculating 
the full Hessian. In Subsection Selection of frequencies we explain how we select the frequencies onto which the Hessian is projected. Finally, we put all pieces together in Subsection The new scheme. For completeness we also present the partial Hessian approach in Subsection Results.

Terminology and definitions

Consider the matrix of second derivatives, also known as Hessian,

$(H)_{i j}=\frac{\partial^{2} E}{\partial R_{i} \partial R_{j}}$.

Here $\mathrm{E}$ is the total energy. The indices $\mathrm{i}$ and $\mathrm{j}$ run from 1 to $3 \mathrm{~N}$, where $\mathrm{N}$ is the number of atoms in the system. Each of the $R_{i}$ represents the displacement of one atom in x, y or $\mathrm{z}$ direction. For example, $R_{1}$ represents displacement of the first atom in x direction, $R_{2}$ represents displacement of the first atom in y direction and so on. The Hessian as in Eq. 3 is expressed in cartesian basis. It is a $3 \mathrm{~N} \times 3 \mathrm{~N}$ matrix. We can find the eigenvectors and eigenvalues according to

$H \cdot \mathrm{q}_{i}=\lambda_{i} \mathrm{q}_{i}$

The $\mathbf{q}_{i}$ are the eigenvectors and $\lambda_{i}$ are the eigenvalues. Since the Hessian is symmetric it is guaranteed that we find $3 \mathrm{~N}$ pairs of eigenvectors and eigenfunctions.

From now on we will call the eigenvectors, $\left\{\mathbf{q}_{i}\right\}$ vibrations of the molecule. The vibrations show the movement of the molecule. They can be visualized by standard visualization software. Each vibration is associated with a wave number, which is calculated from the eigenvalue, $\lambda_{i}$. The wave number is a measure for the energy of that particular vibration. The pair of vibration and associated wave number we will call frequencies.

\section{Projected Hessian}

In this Subsection we consider projected Hessians. Let us assume we have a set of $\mathrm{M}$ orthonormal vectors $\left\{\mathbf{b}_{i}\right\}$. We collect the vectors in a matrix $B$. The projection of the Hessian, $H$, onto the space spanned by the vectors $\mathbf{b}_{i}$ results in the projected Hessian $\widetilde{H}$. It is calculated as

$\widetilde{H}=B \cdot B^{T} \cdot H \cdot B \cdot B^{T}$.

The projected Hessian $\widetilde{H}$ is a $\mathrm{M} \times \mathrm{M}$ matrix. Let us examine the special case, where the vectors $\left\{\mathbf{b}_{i}\right\}$ span the complete space and $M=N$. In this case the projected Hessian, $\widetilde{H}$, and the original Hessian, $H$, are identical. Consequently, the eigenvectors and eigenvalues will be identical, too.

Let us now consider the case where $M<3 N$, i.e., the vectors $\left\{\mathbf{b}_{i}\right\}$ do not span the complete space. From the projected Hessian, $\widetilde{H}$, we can calculate its eigenvectors $\widetilde{q}_{i}$ and eigenvalues $\widetilde{\lambda}_{i}$ via Eq. 4. A result of the projection is that $3 \mathrm{~N}-\mathrm{M}$ eigenvalues will be zero. The question is whether (some of) the remaining eigenvalues and corresponding eigenvectors are also eigenvalues and eigenvectors of the original Hessian, $H$. The answer is that all eigenvectors $\mathbf{q}_{i}$ that lie in the space spanned by $B$ will be in the set of $\left\{\widetilde{q}_{i}\right\}$. The corresponding eigenvalues $\widetilde{\lambda}_{i}$ will be identical to the eigenvalues $\lambda_{i}$. The remaining $\widetilde{q}_{i}$ and $\widetilde{\lambda}_{i}$ are only approximations to the exact $\mathbf{q}_{i}$ and $\lambda_{i}$.

\section{Calculation of selected frequencies}

Let us assume we have a set of $\mathrm{M}$ orthonormal vectors, $\left\{\mathbf{b}_{i}\right\}$. We are interested in eigenvalues of the Hessian projected onto the space spanned by $\left\{\mathbf{b}_{i}\right\}$. The question is how do we practically do this, without first calculating the full Hessian, which appears in Eq. 5.

To this end let us examine the product of the Hessian with one of the vibrations, $H \mathbf{b}_{i}$. The expression for the ath element reads,

$$
\begin{aligned}
\left(H \cdot b_{i}\right)_{a} & =\sum_{j}^{3 N} \frac{\partial^{2} E}{\partial R_{a} \partial R_{j}} b_{i, j} \\
& =\frac{\partial^{2} E}{\partial R_{a} \partial b_{i}} \\
& =\frac{\partial}{\partial b_{i}} \frac{\partial E}{\partial R_{a}}
\end{aligned}
$$

In the above equations $b_{i, j}$ is the jth element of the vector $\mathbf{b}_{i}$, i.e., the displacement of the j-th cartesian coordinate. In Eq. 7 we performed the summation of Eq. 6. Going to Eq. 8 we used the fact that the order of differentiation can be reversed. We obtain an expression that represents the directional derivative of the gradient. In other words, the multiplication $H \cdot \mathbf{b}_{i}$, and hence $H \cdot B$, can be done by the finite difference method. The remaining matrix multiplications in Eq. 6 are straight forward.

Selection of frequencies

In this Subsection we describe how to identify the frequencies that contribute to the isotope factor (IF) in Eq. 2. More precisely, we give an order for the frequencies in which the contribution decreases. We assume in this Subsection that we have the vibrations, $q_{i}^{L}$, and wave numbers, $v_{i}^{L}$, of the light isotopologe. Likewise we have the vibrations, $q_{i}^{H}$, and wave numbers, $v_{i}^{H}$, of the heavy isotopologe. In a first step, we match the vibrations of 
the two isotopologes. This is done by calculating the overlap $\left\langle q_{i}^{L}\right| q_{i}^{H}>$ and pairing those vibrations which show the largest overlap. From now on we assume that the index $\mathrm{i}$ is chosen such that the $\mathrm{i}$-th vibration of the light isotopologe, $q_{i}^{L}$, has largest overlap with the ith vibration of the heavy isotopologe, $q_{i}^{H}$.

In a second step we evaluate the contribution of each of the vibration pairs to the isotope factor. Please recall that

$I F\left(\left\{u_{i, L}\right\},\left\{u_{i, H}\right\}\right)=\Pi \frac{v_{i, H} \cdot \sinh \left[v_{i, L} h /(2 k T)\right]}{v_{i, L} \cdot \sinh \left[v_{i, H} h /(2 k T)\right]}$.

We calculate the contribution of each vibration pair to the isotope factor by

$I F_{i}=\frac{v_{i, H} \cdot \sinh \left[v_{i, L} h /(2 k T)\right]}{v_{i, L} \cdot \sinh \left[v_{i, H} h /(2 k T)\right]}$.

Many of the contributions, $I F_{i}$, will be one, and hence will not contribute to the isotope factor. The rest of the vibration pairs will fall into two groups. Some will have a contribution $I F_{i}$ larger than one. Some of them will have a contribution $I F_{i}$ smaller than one.

We can now make three lists of vibration pairs. First, we have the lists of vibration pairs that give a contribution $I F_{i}$ smaller than one. Within this list we order the vibration pairs by ascending $I F_{i}$. Then there is a list of vibrations with contribution $I F_{i}$ larger than one. Within this list we order the vibration pairs by descending $I F_{i}$. Finally, we have a list of vibration pairs that do not contribute at all.

\section{The new scheme}

In this Subsection we explain how the new scheme works. Assume we have a system, whose geometry has been optimized to a minimum with a given level of theory. The level of theory we will call target theory (TT). The goal is now to obtain the isotope factor for this system at the TT. First, we choose a lower level of theory (LL). We calculate the Hessian of our system at LL in the geometry optimized at TT. We are fully aware, that the frequencies calculated from this Hessian are not physical, since the Hessian is evaluated at a non-stationary point. This does not mean much in our case, since the Hessian is used only to obtain an orthonormal set of vibrations, regardless if they have a physical interpretation or not.

From the Hessian calculated at LL we calculate the vibrations and wave numbers. According to the procedure described in Subsection Selection of frequencies we obtain three lists of LL vibrations. We discard the list of LL vibrations that do not contribute to the LL isotope factor (IF). Typically, the list with LL vibrations that contribute a factor of smaller than one is short. The list with LL vibrations that contribute a factor of larger than one is much longer.
We now use the LL vibrations obtained in the previous step. First we choose all LL vibrations from the short list, which contribute a factor smaller than one. We calculate their directional derivative as described in Subsection Calculation of selected frequencies. Subsequently, we calculate the approximate TT vibrations and approximate TT wave numbers according to Section Projected Hessian. From the approximate TT wave numbers we can calculate the approximate TT isotope factor. Now we start an iteration. The first LL vibration from the long list, which contributes a factor larger than one, is added to the set of orthonormal vectors $\left\{\mathbf{b}_{i}\right\}$. Its directional derivative is calculated according to Subsection Calculation of selected frequencies. The directional derivative is joined with the previously calculated directional derivatives. The approximate TT vibrations and wave numbers are calculated. We obtain an additional approximate TT vibration and approximate TT wave number, while the previous approximate TT vibrations and approximate TT wave numbers change. We calculate the new approximate TT isotope factor. We continue this iteration until convergence of the approximate TT IF to the desired accuracy is reached.

The set-up of our scheme has two advantages. Firstly, it will inevitably converge to the correct result. If we feed all LL vibrations to the algorithm of Subsection Calculation of selected frequencies we will obtain the exact TT vibrations with exact TT wave numbers. This is because all LL vibrations form a complete orthonormal basis. Consequently, the projection performed in Eq. 5 will leave the Hessian unaltered. Secondly, our scheme gives a lower bound to the TT isotope factor at each step. This is because we first include all LL vibrations that give a factor of less than one. The remaining LL vibrations will only yield larger TT isotope factors.

\section{Partial Hessian approach}

In the partial Hessian approach the approximate wave numbers are calculated according to Subsections Projected Hessian and Calculation of selected frequencies. The vectors $\mathbf{b}_{i}$ are chosen to be unit vectors with zeros everywhere but one entry is one. Per atom there are three vectors $\mathbf{b}_{i}$. Each of the three represents a displacement of the chosen atom in $\mathrm{x}, \mathrm{y}$ or $\mathrm{z}$ direction. For example, choosing the jth atom means inclusion of the vectors with a one at position $3 \cdot(j-1)+1,3 \cdot(j-1)+2$ and $3 \cdot(j-1)+3$.

\section{Results}

We applied our scheme to oxamate bound in the active site of lactate dehydrogenase (LDH) from the rabbit muscle (PDB ID 3H3F1). The system is shown schematically in 
Fig. 1. It contains 139 atoms. Oxamate inhibits the LDH activity to catalyze the reversible conversion of pyruvate to lactate in the presence of nicotinamide adenine dinucleotide NADH cofactor. The full system has previously been investigated [17-19].

For the calculations of the isotope factor (IF) we labelled the two carboxylic oxygen atoms (O1 and $\mathrm{O} 2$ in Fig. 1) of the oxamate with the isotope ${ }^{18} \mathrm{O}$. We performed a geometry minimization at $\mathrm{B} 3 \mathrm{LYP} / 6-31 \mathrm{G}^{*}$ level of theory [20-23]. This level represents our target theory (TT). For comparison we also calculated the full Hessian at the optimized geometry. We chose AM1 [24-26] as our low level of theory (LL). We calculated the full Hessian with AM1 in the geometry optimized with B3LYP/6-31G*. We used ISOEFF [27] to match the LL vibrations as described in Subsection Selection of frequencies. The AKIRA [28] code was used to perform the algorithm described in Subsection Projected Hessian and Calculation of selected frequencies. All electronic structure calculations were performed with Gaussian [29].
Results from the exact frequencies

First we analyze how many TT frequencies are necessary to obtain the correct IF. We use the TT Hessian for the analysis described in Subsection Selection of frequencies. This represents the ideal case. We know already all TT vibrations and TT wave numbers. We only put them in order and calculate the IF for a subset of TT wave numbers. Table 1 shows the results of oxamate in the active site of LDH. The first column shows the number of TT wave numbers. The second column shows the error in $\%$ to the correct IF value of 1.209. In total there are 411 frequencies in this system.

We see that the IF indeed converges from below. For an accuracy of $-7 \%$ approximately 20 vibrations are needed. With 60 vibrations we obtain an accuracy of $-1 \%$. With about 150 vibrations the error reduces to $-0.1 \%$. An error of $-0.01 \%$ is achieved with approximately 250 vibrations.

The presented analysis verifies our implementation. Moreover, we see that not all exact vibrations and wave
Fig. 1 Oxamate in the active site of $\mathrm{LDH}$. $\mathrm{O} 1$ and $\mathrm{O} 2$ are labeled with isotope ${ }^{18} \mathrm{O}$ in the isotope factor calculations. The atoms $\mathrm{O} 1$ through $\mathrm{H} 12$ are included in the partial Hessian approach

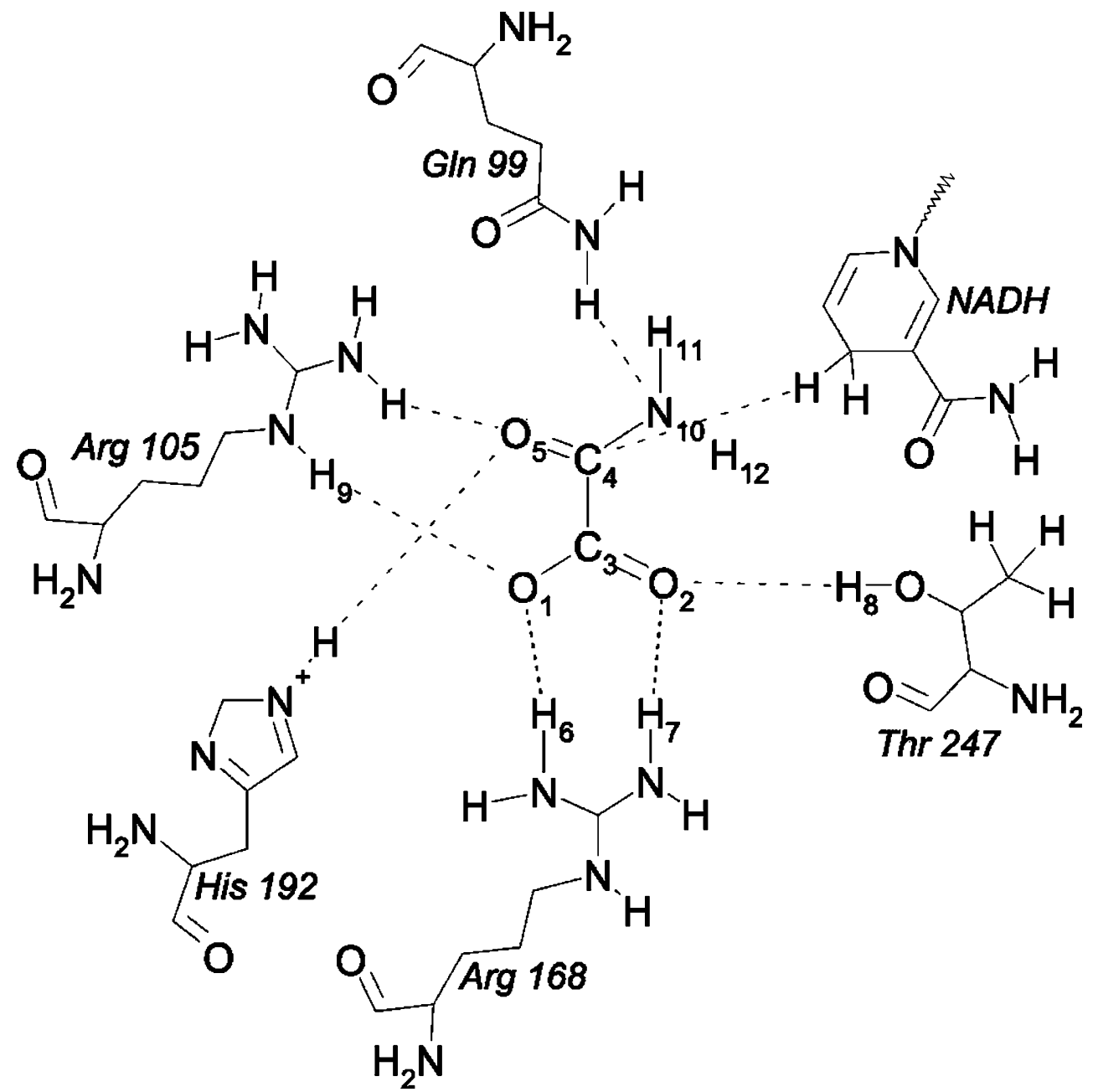


Table 1 Results for various numbers of exact wave numbers of oxamate in the active site of LDH. The second column gives the error in $\%$ of the approximate isotope factor

\begin{tabular}{ll}
\hline Number of vectors & Error in \% \\
\hline 10 & -16.25 \\
20 & -6.87 \\
30 & -2.73 \\
40 & -1.69 \\
50 & -1.25 \\
60 & -0.75 \\
100 & -0.28 \\
150 & -0.10 \\
250 & -0.01 \\
300 & 0.00 \\
\hline
\end{tabular}

numbers are necessary to obtain an accurate IF. An accuracy of $0.1 \%$ usually suffices. Hence, we need to calculate only roughly 150 vibrations and wave numbers for oxamate in LDH. Thus, a full Hessian analysis calculates three times more wave numbers than necessary. We remind the reader, that for the targeted application analytical Hessian evaluation is not possible. Hence, the computational time saved would be roughly a factor of three.

Results from the new scheme

We now turn to a more realistic example. We perform a full Hessian analysis at AM1 level of theory, which will serve as our low level (LL). The calculated LL vibrations and LL wave numbers are ordered according to the procedure described in Subsection Selection of frequencies. We then feed the ordered LL vibrations to our new scheme described in Subsection The new scheme. The results are shown in Table 2.

The first column of Table 2 shows the number of LL vibrations fed into the algorithm described in Subsection The new scheme. The second column gives the error of the IF calculated therefrom. We see the same pattern as in the exact example before. With $20 \mathrm{LL}$ vibrations we obtain an accuracy of $-4 \%$. About $60 \mathrm{LL}$ vibrations are needed to reduce the error to $-1 \%$. For an error of $-0.1 \% 150 \mathrm{LL}$ vibrations are needed. Extending the number of LL vibrations to 250 we reduce the error to below $-0.01 \%$.

Columns three, four, and five give an indication about the accuracy of the wave numbers that are obtained. They give the number of approximate TT wave numbers that differ by less than 10,25 , and $50 \mathrm{~cm}^{-1}$ from the exact TT wave numbers, respectively. We see that a large percentage of the approximate TT wave numbers is in very good agreement with the exact TT wave numbers. For example, from the 150 approximate TT wave numbers 92 differ by less than $10 \mathrm{~cm}^{-1}$. Almost all (130) differ by less than $50 \mathrm{~cm}^{-1}$, which is usually still considered to be in fair agreement. This analysis shows that the accurate IF, that we obtain, is not a mere coincidence. It is accurate because the approximate TT wave numbers are accurate.

\section{Results from the partial Hessian approach}

For comparison we show results from the partial Hessian approach in Table 3. The first column shows the number of basis vectors. They are all multiples of three, since for each chosen atom there are three degrees of freedom. In each line one additional atom is included. The atoms are included in the following order: $\mathrm{O} 1, \mathrm{O} 2, \mathrm{C} 3, \mathrm{C} 4, \mathrm{O} 5, \mathrm{H} 6$, H7, H8, H9, N10, H11, H12. For comparison see also Fig. 1.

The results are surprising, if we recall the large amount of wave numbers needed to obtain an accurate IF (cf. Table 1). Including only one of the labeled atoms (first row of Table 3) the error is roughly $-8 \%$. Adding the second labeled oxygen atom (second row of Table 3 ) reduces the error to only $2.4 \%$. In comparison, there are more than 30 exact TT wave numbers needed to obtain this accuracy (see Table 1). Including the carbon atom that is linked to both labeled oxygen atoms in the partial Hessian reduces the error by an order of magnitude down to $0.2 \%$. We see that inclusion of only the carboxyl group of oxamate, i.e., calculation of nine approximate wave numbers, gives us an accuracy equivalent to including more than 100 exact wave numbers (cf. Table 1). What is even more surprising is that the approximate wave numbers calculated from only the atoms of the carboxyl group are rather inaccurate. Only one

Table 2 Results for various numbers of approximate wave numbers obtained from the new scheme for oxamate in LDH. The second column gives the error in $\%$ of the approximate isotope factor. The last three columns give the number of wave numbers that show an error of less than 10,25 and $50 \mathrm{~cm}^{-1}$, respectively

\begin{tabular}{lllll}
\hline $\begin{array}{l}\text { Number of } \\
\text { vectors }\end{array}$ & Error in \% & $<10 \mathrm{~cm}^{-1}$ & $<25 \mathrm{~cm}^{-1}$ & $<50 \mathrm{~cm}^{-1}$ \\
\hline 10 & -12.80 & 2 & 6 & 8 \\
20 & -3.95 & 5 & 10 & 19 \\
30 & -2.27 & 8 & 21 & 26 \\
40 & -1.59 & 10 & 27 & 36 \\
50 & -1.25 & 17 & 33 & 45 \\
60 & -0.82 & 22 & 43 & 53 \\
100 & -0.40 & 55 & 80 & 89 \\
150 & -0.10 & 92 & 120 & 130 \\
250 & 0.00 & 166 & 206 & 223 \\
300 & 0.00 & 247 & 273 & 278 \\
\hline
\end{tabular}


Table 3 Results for various numbers of approximate wave numbers obtained from the partial Hessian approach for oxamate in LDH. The second column gives the error in \% of the approximate isotope factor. The last three columns give the number of wave numbers that show an error of less than 10,25 and $50 \mathrm{~cm}^{-1}$, respectively

\begin{tabular}{lllll}
\hline $\begin{array}{l}\text { Number of } \\
\text { vectors }\end{array}$ & Error & $<10 \mathrm{~cm}^{-1}$ & $<25 \mathrm{~cm}^{-1}$ & $<50 \mathrm{~cm}^{-1}$ \\
\hline 3 & -7.78 & 0 & 0 & 0 \\
6 & 2.41 & 0 & 0 & 1 \\
9 & 0.20 & 1 & 3 & 4 \\
12 & 0.14 & 1 & 3 & 4 \\
15 & 0.13 & 1 & 2 & 5 \\
18 & 0.10 & 1 & 2 & 5 \\
21 & 0.06 & 1 & 2 & 7 \\
24 & 0.04 & 1 & 2 & 7 \\
27 & 0.04 & 1 & 2 & 7 \\
30 & 0.03 & 1 & 5 & 9 \\
33 & 0.02 & 5 & 8 & 12 \\
36 & 0.02 & 17 & 20 & 22 \\
\hline
\end{tabular}

of the nine approximate wave numbers is accurate to within $10 \mathrm{~cm}^{-1}$ and only four are accurate to within $50 \mathrm{~cm}^{-1}$.

Inclusion of the carbonyl group ( $\mathrm{C} 4$ and $\mathrm{O} 5)$ and the four hydrogen atoms that form a hydrogen bond with the oxygens of the carboxyl group (H6, H7, H8 and H9) reduces the error by another order of magnitude. With 27 approximate wave numbers we obtain an error of $0.04 \%$ (compare Table 3). While the accuracy of the IF increases the accuracy of the wave numbers does not. There is still only one wave number accurate to within $10 \mathrm{~cm}^{-1}$ and only seven to within $50 \mathrm{~cm}^{-1}$.

Including the remaining amine group of oxamate does not change the error (compare last row in Table 3). The number of approximate wave numbers that are in agreement with the exact wave numbers rises to 17 (error less than $10 \mathrm{~cm}^{-1}$ ) and 22 (error less than $50 \mathrm{~cm}^{-1}$ ). The accuracy of the wave numbers may not be so surprising. Oxamate is not covalently bound to the rest, so that the vibrations are rather localized at oxamate. Consequently, we recover almost all vibrations of oxamate (17 out of 18). Nevertheless, it is surprising to see that only 24 approximate wave numbers suffice to obtain an accuracy, which is achieved only with 200 exact wave numbers (cf. Table 1).

One may argue, that it is not the absolute value of the wave numbers that is of importance for the IF. Rather the shift that comes with substituting one isotope with another is important to recover the correct IF. This is, of course, true only to some extent. A too large error in the absolute wave numbers must yield an incorrect IF even if the shift is reproduced correctly. However, for small absolute errors this is certainly true. In particular, it has been shown [30] that the Bigeleisen-Mayer equation (cf. Eq. 1) is insensitive to small errors in the absolute wave numbers. However, this analysis was performed including all wave numbers. In the case of the partial Hessian approach only a few wave numbers are calculated. Indeed, we must conclude that there is a large error on the calculated shifts. If there was no error, we would not recover the correct IF, because 36 accurate wave numbers are not enough (cf. Table 1).

The reader might wonder whether the presented results are general. Indeed, we find the same picture for different situations. We have performed the same analysis labeling different atoms in oxamate or even in the residues. In each case we find the same overall picture. Analysis of the exact TT wave numbers suggests that many (more than 100) wave numbers are needed to calculate the IF. Applying our scheme we accurately reproduce the IF with roughly the same number of approximate TT wave numbers. However, the partial Hessian approach gives a highly accurate IF with only a few (around 25) approximate wave numbers.

\section{Conclusions}

In this paper we suggest a new scheme to calculate the isotope effect. We examine the scheme by calculating the isotope factor of oxamate bound in the active site of lactate dehydrogenase with approximate wave numbers. We compare our results to the isotope factor calculated from all exact wave numbers at B3LYP/6-31G*. We show that we need equally many approximate wave numbers to obtain a given accuracy, as exact wave numbers are needed (cf. Table 2). We show that our new scheme converges from below, which indicates that the calculated isotope factor is always a lower bound. We also show that the approximate wave numbers that we calculate are in very good agreement with the exact wave numbers, proving that the accurate isotope factor is not a mere coincidence. For the shown system our scheme reduces the computational cost by a factor of approximately three, when compared to an evaluation of the Hessian by finite differences.

We also show results obtained by the partial Hessian approach. The results suggest that it suffices to calculate the partial Hessian for only a very small number of atoms, including the labeled atoms (cf. Table 3). While these results are in line with previous findings $[5,31]$ they seem to contradict the analysis of the exact wave numbers (cf. Table 1), which suggest that a large number of wave numbers need to be calculated to obtain an accurate isotope factor. At this moment we are not able to give an explanation for the accuracy of the partial Hessian approach. 
Acknowledgements ADD acknowledges funding from the European Community's Seventh Framework Program (FP7) under the grant agreement no 212781 and access to supercomputing facilities at Cyfronet (Poland). DR acknowledges financial support from the German Research Foundation (DFG) under grant no. RO 3894/1- 1. Useful discussions with Prof. Piotr Paneth and Prof. Vicente Moliner are gratefully acknowledged. The authors would like to thank Dr. Victor Anisimov for the implementation of additional features in ISOEFF.

Open Access This article is distributed under the terms of the Creative Commons Attribution Noncommercial License which permits any noncommercial use, distribution, and reproduction in any medium, provided the original author(s) and source are credited.

\section{References}

1. Kohen A, Limbach H-H (2006) Isotope effects in chemistry and biology. Taylor and Francis, New York

2. Schramm VL (2007) Curr Opin Chem Biol 11:529

3. Wolfsberg M, Van Hook WA, Paneth P (2010) Isotope effects. Springer, New York

4. Bigeleisen J, Goeppert-Mayer M (1947) J Chem Phys 15:261

5. Wolfsberg M, Stern MJ (1964) Pure Appl Chem 8:225

6. Sims LB, Lewis DE (1984) In: Buncel E, Lee CC (eds) Isotopes in organic chemistry, vol. 6. Elsevier, Amsterdam, p 162259

7. Stern MJ, Wolfsberg M (1966) J Chem Phys 45:4105

8. Sims LB, Burton GW, Lewis DE (1997) BEBOVIB-IV, QCPE No. 337 (Quantum Chemistry Program Exchange, Department of Chemistry, University of Indiana: Bloomington, IN,)
9. Rodgers J, Femac DA, Schowen RL (1982) J Am Chem Soc 104:3263

10. Casamassina TE, Huskey WP (1993) J Am Chem Soc 115:14

11. Berti PJ (1999) Meth Enzymol 308:355

12. Paneth P (1995) Comput Chem 19:231

13. Saunders WH (2001) CCACAA 74:575

14. Dybala-Defratyka A, Rostkowski M, Matsson O, Westaway CK, Paneth P (2004) J Org Chem 69:4900

15. Redlic O (1935) Z Phys Chem B 28:371

16. Bigeleisen J (1949) J Chem Phys 17:675

17. Gawlita E, Anderson VE, Paneth P (1994) Eur Biophys J 23:353

18. Świderek K, Panczakiewicz A, Bujacz A, Bujacz G, Paneth P (2009) J Phys Chem B 113:12782

19. Świderek K, Paneth P (2010) J Phys Chem B 114:3393

20. Becke AD (1996) J Chem Phys 98:5648

21. Lee C, Yang W, Parr RT (1988) Phys Rev B 37:785

22. Stephens PJ, Devlin FJ, Chabalowski CF, Frisch MJ (1994) J Phys Chem 98:11623

23. Hehre WJ, Ditchfield R, Pople JA (1972) J Chem Phys 56:2257

24. Dewar MJS, Zoebisch EG, Healy EF, Stewart JJP (1985) J Am Chem Soc 107:3902

25. Dewar MJS, Jie CJ (1989) J Mol Struct 187:1

26. Dewar MJS, Yuan YC (1990) Inorg Chem 29:3881

27. Anisimov V, Paneth P (1999) J Math Chem 26:75

28. Reiher M, Neugebauer J (2003) J Chem Phys 118:1634

29. Frisch MJ et al (2003) Gaussian 03 (Gaussian, Inc., Wallingford, $\mathrm{CT}$, )

30. Schaad LJ, Bytautas L, Houk KN (1999) Can J Chem 77:875

31. Ruggiero GD, Guy SJ, Marti S, Moliner V, Williams IH (2004) J Phys Org Chem 17:592 